\title{
Origem da artéria cerebelar inferoposterior sobre o arco posterior de $\mathrm{C} 1^{*}$
}

\section{Origin of the Posterior Inferior Cerebellar Artery over the C1 Posterior Arch}

\author{
Zeferino Demartini Júnior ${ }^{1}$ () Ricardo Munhoz da Rocha Guimarães ${ }^{2}$ Emiliano Neves Vialle ${ }^{1}$ \\ Gelson Luis Koppe ${ }^{1}$ Alexandre Novicki Francisco ${ }^{1}$ Luana Antunes Maranha Gatto ${ }^{1}$ \\ ${ }^{1}$ Departamento de Neurocirurgia, Pontifícia Universidade Católica do \\ Paraná (PUC-PR), Curitiba, PR, Brasil \\ 2 Departamento de Ortopedia, Pontifícia Universidade Católica do \\ Paraná (PUC-PR), Curitiba, PR, Brasil \\ Endereço para correspondência Zeferino Demartini Júnior, \\ Departamento de Neurocirurgia, Pontifícia Universidade Católica do \\ Paraná (PUC-PR), Curitiba, PR, Brasil (e-mail: demartiniz@gmail.com).
}

Rev Bras Ortop 2019;54:605-608.

\section{Resumo \\ Palavras-chave \\ - artéria vertebral \\ - angiografia cerebral \\ - variação anatômica \\ - síndrome medular lateral \\ - complicações intraoperatórias}

Abstract
A artéria cerebelar inferoposterior geralmente nasce do segmento intracraniano (V4) da artéria vertebral. Apesar de ter diâmetro médio de $2 \mathrm{~mm}$, usualmente irriga áreas eloquentes do encéfalo. Quando ocluída, seja por trauma ou cirurgia, pode causar infarto no tronco encefálico e no cerebelo. Apresentamos um caso de artéria cerebelar inferoposterior com origem anômala no segmento cervical (V3) da artéria vertebral, demonstrado por angiografia. Os resultados foram registrados e comparados com os de publicações anteriores. Foram fornecidas breves explicações sobre a anatomia, anomalias vasculares e embriologia. A revisão da literatura mostrou que os ramos anômalos do segmento cervical da artéria vertebral são infrequentes e devem ser conhecidos. Uma melhor compreensão da anatomia e suas variações permite fazer um diagnóstico topográfico preciso, bem como planejar a abordagem e a terapia cirúrgicas ideais. O conhecimento dessa variação anatômica é essencial, pois, se confundida com um ramo muscular e coagulada, pode causar isquemia com sequelas incapacitantes.

The posterior inferior cerebellar artery usually arises from the intracranial segment (V4) of the vertebral artery. Despite its mean diameter of $2 \mathrm{~mm}$, it usually irrigates important areas of the brain. When occluded, whether due to trauma or surgery, it may cause infarction in the brain stem and cerebellum. The present report describes a case of incidental finding of a posterior inferior cerebellar artery arising from the

\footnotetext{
Trabalho desenvolvido no Hospital Universitário Cajuru, Pontifícia Universidade Católica do Paraná (PUC-PR), Curitiba, PR, Brasil. Publicado Originalmente por Elsevier Editora Ltda.
}

recebido

03 de Outubro de 2017 aceito

21 de Dezembro de 2019
DOI https://doi.org/

10.1016/j.rbo.2017.12.025. ISSN 0102-3616.
Copyright $\odot 2019$ by Sociedade Brasileira License terms de Ortopedia e Traumatologia. Published by Thieme Revnter Publicações Ltda, Rio de Janeiro, Brazil 


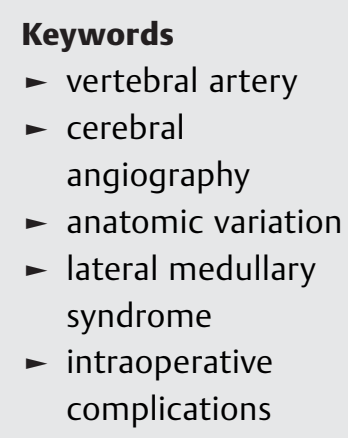

cervical segment (V3) of the vertebral artery, demonstrated by angiography. The findings were recorded and compared to those of earlier publications. Brief explanations regarding anatomy, vascular anomalies and embryology were provided. A literature review showed that anomalous branches of the cervical segment of the vertebral artery are infrequent and must be known. A better understanding of anatomy and its variations enables an accurate topographic diagnosis, as well as the planning of the optimal surgical approach and therapy. Knowledge of this anatomical variation is essential because, if it is mistaken for a muscle branch and coagulated, this can cause ischemia and disabling sequelae.

\section{Introdução}

A artéria cerebelar inferoposterior (ACIP), chamada na Nomina Anatomica de arteria inferior posterior cerebelli, usualmente nasce do segmento intracraniano (V4) da artéria vertebral, e irriga áreas vitais do encéfalo, em especial do tronco encefálico. ${ }^{1,2}$ Sua oclusão inadvertida durante uma cirurgia pode causar um infarto que, apesar de volumetricamente pequeno, produz sequelas graves e incapacitantes. ${ }^{3} \mathrm{~A}$ variação anatômica pode predispor à lesão iatrogênica mesmo com a técnica cirúrgica adequada. ${ }^{4}$ Apresentamos um caso de ACIP com origem anômala no segmento cervical (V3) da artéria vertebral que ilustra essa possibilidade.

\section{Relato do caso}

Paciente do sexo masculino, de 51 anos, deu entrada no setor de emergência com queixa de cefaleia intensa de início súbito. Ele foi submetido a tomografia de crânio que evidenciou hemorragia intracraniana em espaço espaço subaracnoide, e suspeitou-se de etiologia aneurismática. Em seguida, foi submetido a angiografia por subtração digital dos vasos cervicais e cranianos, que excluiu a presença de aneurisma ou malformação arteriovenosa. Durante o exame de angiografia, foi identificada variação anatômica da ACIP esquerda, a qual tinha origem anômala no segmento cervical (V3) da artéria vertebral ipsilateral ( - Fig. 1 ). O paciente apresentou melhora clínica e recebeu alta assintomático após sete dias de internamento e depois de ter repetição da angiografia.

\section{Discussão}

A ACIP pode ser considerada a artéria que irriga a parte inferoposterior do cerebelo, mas geralmente é definida como a artéria cerebelar que surge da artéria vertebral: é geralmente o maior ramo dessa artéria, e um dos três principais nutridores do cerebelo. ${ }^{1,2,4}$ Em estudo anatômico, Lister et al ${ }^{1}$ identificaram a origem típica dessa artéria no segmento V4 da artéria vertebral na maioria dos casos; portanto, ela tem localização intracraniana e intradural, está em média $8 \mathrm{~mm}$ acima do forame magno, e $17 \mathrm{~mm}$ abaixo da junção vertebrobasilar. ${ }^{2,4} \mathrm{O}$ início da ACIP encontra-se próximo da oliva inferior do tronco encefálico, e circunda e irriga as porções anterior, lateral ou posterior do bulbo através de ramos perfurantes diretos ou circunflexos. ${ }^{1}$ O segmento distal da ACIP tem diversas variações descritas, mas essas não têm relação com a instrumentação cervical superior. ${ }^{4} \mathrm{Em}$ geral, após cruzar anteriormente os nervos cranianos baixos (glossofaríngeo, vago, acessório e hipoglosso), a ACIP se dirige posteriormente, percorre as tonsilas cerebelares, e dá ramos que se distribuem para o plexo coroide do quarto ventrículo, para metade do vermis, e para a superfície suboccipital do hemisfério cerebelar. ${ }^{1}$

O diâmetro da ACIP varia de 0,5 a 3,4 mm, com média de $2 \mathrm{~mm}{ }^{1}$ No entanto, apesar de seu diminuto calibre, sua oclusão pode trazer graves sequelas por irrigar áreas vitais do encéfalo. As consequências variam de uma lesão isquêmica clinicamente silenciosa até infarto de porções do tronco encefálico ou cerebelo, com edema, hemorragia e morte. ${ }^{1}$ Praticamente todas as oclusões de ACIP resultam em infarto, enquanto pouco mais de metade das oclusões de artéria vertebral resultam em infarto, uma vez que pode haver fluxo colateral através da artéria vertebral contralateral. ${ }^{1}$ A oclusão da ACIP causa infarto na porção lateral do bulbo, dorsal ao núcleo olivar inferior, e resulta na síndrome bulbar lateral, descrita em 1895 por Wallenberg ${ }^{3}$ em análise post-mortem. A síndrome de Wallenberg inclui: termoanalgesia no hemicorpo contralateral à lesão, por lesão do trato espinotalâmico lateral bulbar; hemi-hipo/anestesia facial ipsilateral, por lesão do núcleo e trato espinhal do trigêmeo $(\mathrm{V})$; disfagia e disfonia, por lesão do núcleo ambíguo que afeta funções dos nervos vago (X) e glossofaríngeo (IX); síndrome vestibular ipsilateral, com vertigem rotatória, diplopia, lateropulsão e nistagmo, por lesão de núcleos vestibulares; síndrome de Horner ipsilateral, por lesão de fibras descendentes simpáticas; e síndrome cerebelar atáxica ipsilateral, por lesão do pedúnculo cerebelar inferior. ${ }^{3}$ A ACIP tem o mais complexo, tortuoso e variável curso e área de irrigação das artérias cerebelares. ${ }^{1,2} \mathrm{~A}$ incidência de origem extradural dessa artéria varia na literatura de 0,7 a $20 \%^{2,5}$ Ramos musculares se originam da porção horizontal do segmento V3 da artéria vertebral, e variam em diâmetro de 0,2 a $0,4 \mathrm{~mm}$; podem, portanto, ser confundidos com a ACIP, e favorecem uma lesão iatrogênica. ${ }^{2}$ Ainda assim, a ACIP com origem extradural geralmente se origina na parede lateral ou posterior da artéria vertebral, e tem curso paralelo a essa artéria, o que permite sua identificação (-Fig. 2). ${ }^{2}$ Além disso, ela perfura a dura-máter em contato com a artéria vertebral entre o atlas e o forame magno, mas eventualmente pode penetrar no nível de $\mathrm{C} 1$ ou $\mathrm{C} 2{ }^{2}{ }^{2}$ Reconhecer essa variação anatômica é de 


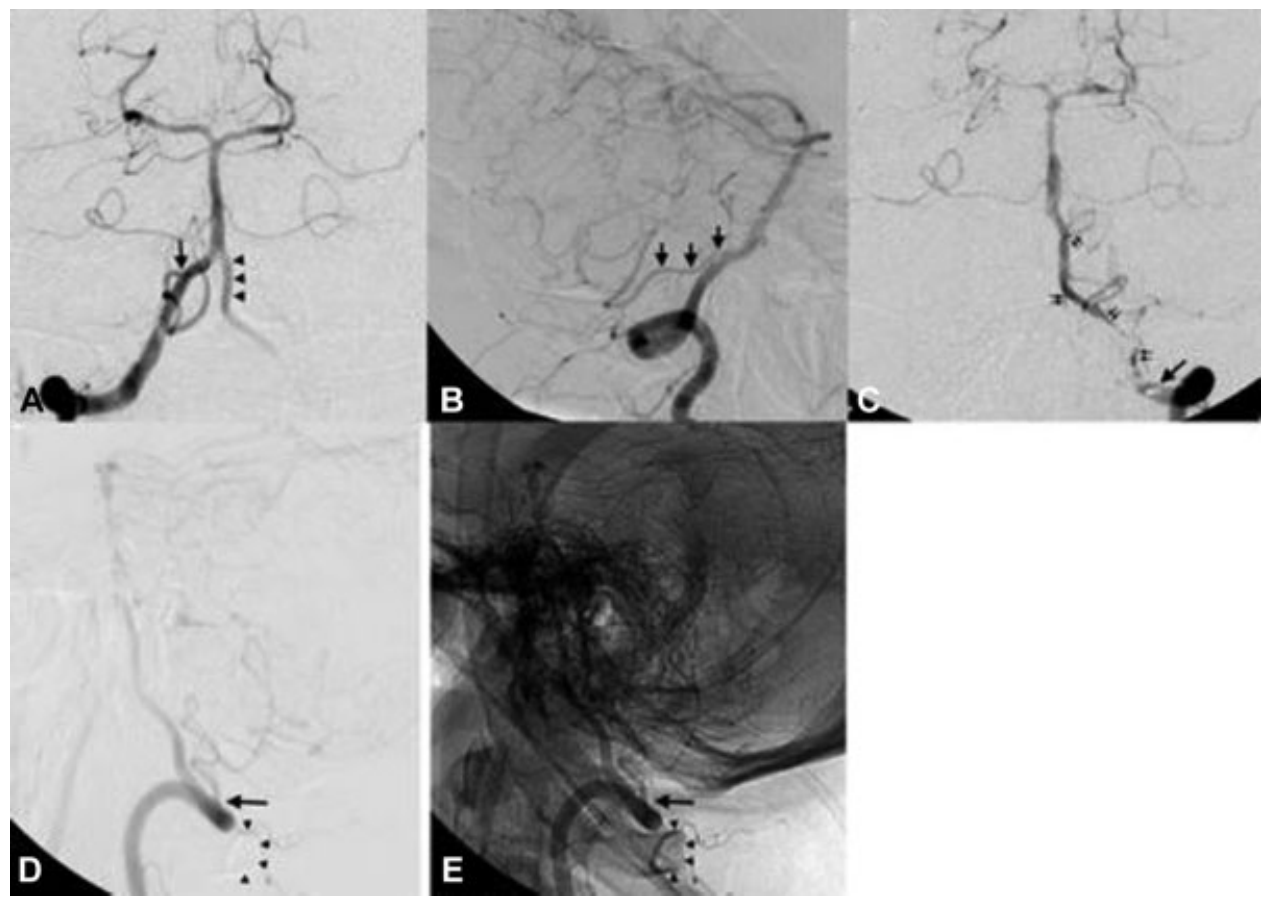

Fig. 1 Imagem de angiografia seletiva da artéria vertebral direita em AP (A) e P (B) mostra a origem normal da artéria cerebelar inferoposterior (setas) no segmento intracraniano (V4) ipsilateral; o segmento V4 contralateral (pontas das setas) não origina a artéria usualmente vista no segmento. Durante injeção seletiva de contraste na artéria vertebral esquerda em AP (C) e P (D) com subtração digital e com referencial anatômico (E), é evidenciada a origem extracraniana da artéria cerebelar inferoposterior esquerda (setas) no segmento cervical (V3), sobre o arco de C1 (pontas das setas), com trajeto paralelo à artéria vertebral (setas duplas). Abreviações: AP, anteroposterior; P, perfil.

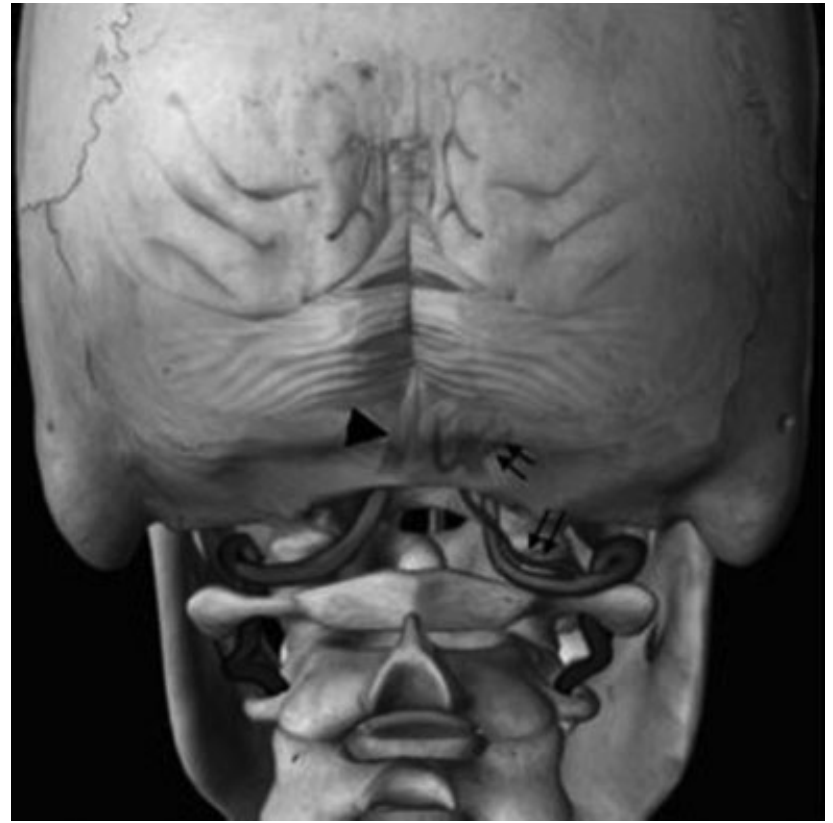

Fig. 2 Imagem que ilustra a relação anatômica entre as vértebras cervicais altas e as artérias vertebrais, as quais penetram na duramáter entre o arco posterior de $\mathrm{C} 1$ e o forame magno. A zona de segurança usualmente se estende até $8 \mathrm{~mm}$ de cada lado da linha média. A artéria cerebelar inferoposterior usualmente se origina no segmento V4 intracraniano (ponta da seta); no caso apresentado, ela se origina sobre o arco de C1 (setas duplas).

fundamental importância em todos os procedimentos invasivos da transição craniocervical, que variam desde uma punção liquórica no nível de C1-C2 até acessos cirúrgicos mais amplos, como os cervicais altos, suboccipital lateral e extremolateral. ${ }^{2,5}$

A fixação e descompressão da junção occipitocervical com parafusos de massa lateral em C1 é considerada segura, e é amplamente usada. ${ }^{4}$ Frequentemente, 0 arco posterior do atlas é removido como parte do procedimento, e, na maioria das vezes, a artéria vertebral está lateralizada e visível; como o espaço para a medula é amplo, pode haver pouca preocupação com a dissecção. As complicações, apesar de relativamente infrequentes, podem ser catastróficas, e seu risco aumenta com a presença de variações anatômicas. ${ }^{4}$ A presença destas variações pode trazer complicações inesperadas, como descrito por Nassr et $\mathrm{al}^{4}$ em um caso de fratura patológica por mieloma múltiplo tratada com fixação occipito-C6, inclusive com colocação de parafuso em massa lateral de C1. O paciente apresentou disartria, desequilíbrio e disdiadococinesia pós-operatórias, e exames de imagem mostraram infarto em território da ACIP; e, apesar do posicionamento adequado do parafuso, havia variante anatômica com trajeto extracraniano da artéria em volta de C1. Uma revisão de 29 casos de infarto da porção lateral do bulbo, inclusive lesão de ACIP, evidenciou sintomas de disfagia, disartria, ataxia, dismetria, disdiadococinesia, dor facial, vertigem, nistagmo, síndrome de Horner, diplopia e mioclonia. ${ }^{6}$ Ainda que nem todos os sintomas se correlacionem perfeitamente com a síndrome bulbar lateral clássica, é necessário um alto índice de suspeição para o diagnóstico, até porque a monitoração neurofisiológica pode não mostrar alterações transoperatórias. ${ }^{4}$ Em outra recente revisão sistemática da literatura, Akinduro et al ${ }^{7}$ encontraram uma incidência de lesões vasculares de 2,9\% durante 
instrumentação atlantoaxial posterior, dos quais $10 \%$ resultaram em infarto ipsilateral, e 6,7\%, em óbito. Tratamento endovascular foi feito em $13,3 \%$, e resultou em reparo da lesão sem déficits permanentes.

O conhecimento pré-operatório de variações anatômicas pode afetar o planejamento cirúrgico e evitar complicações vasculares. ${ }^{4} \mathrm{O}$ maior estudo referente à origem anormal da ACIP abaixo do arco posterior do atlas mostrou uma incidência de $1 \%$ após análise de 346 angiogramas, e foi feito após um caso de lesão da ACIP durante uma punção $\mathrm{C} 1-\mathrm{C} 2 .^{8}$ Múltiplas variações do sistema vertebrobasilar foram descritas e relacionadas a maior risco em cirurgias cervicais altas. ${ }^{4}$ Ectasia vertebral pode ser secundária a dilatação por aterosclerose ou malformação congênita, ${ }^{9}$ e, ao perfurar a dura-máter no espaço interlaminar, pode causar efeito de massa. ${ }^{4}$ Dessa forma, em casos de pacientes com sintomas de neuralgia occipital, inclusive cefaleia pulsátil e imagem de alargamento foraminal, deve haver suspeita de variação anatômica. ${ }^{10} \mathrm{Na}$ variação mais comum, a artéria vertebral ascende lateralmente após deixar o forame transverso de $\mathrm{C} 2$, e entra no canal ósseo criado entre o atlas e o osso occipital. ${ }^{4}$ A próxima variação ocorre quando a artéria vertebral entra no canal medular abaixo do arco posterior de C1 e cruza a superfície da massa lateral. ${ }^{4}$ Por fim, complicações cirúrgicas podem ser causadas pela presença de uma artéria proatlantal, que é uma persistência das anastomoses carotidobasilares embrionárias. ${ }^{4}$ Essa artéria se junta com a artéria vertebral no nível de C1-C2 para entrar no forame magno, e geralmente se associa com hipoplasia ou agenesia da artéria vertebral ipsilateral. ${ }^{4}$ Tokuda et $\mathrm{al}^{5}$ estudaram angiografias de 300 pacientes, e encontraram 2 casos de artéria vertebral que penetrou a dura-máter abaixo de $\mathrm{C} 1,3$ casos de duplicação de artéria vertebral, e 2 casos de ACIP originada em C2. Wang et $\mathrm{al}^{11}$ investigaram 36 pacientes com diagnóstico de occipitalização do atlas, e evidenciaram que $86 \%$ das artérias estariam sob risco de lesão iatrogênica na colocação de parafuso em C1.

A indicação de exames de imagem vasculares pré-operatórios ainda é tema de debate, mas é possível avaliar a localização das artérias por angiotomografia. ${ }^{4}$ Se a superfície anterior da massa lateral de C1 não for perfurada com broca ou fio guia e $o$ parafuso for implantado monocorticalmente, não ocorrerá lesão carotídea. ${ }^{4}$ No entanto, algumas variações anatômicas, inclusive ACIP aberrante e artéria proatlantal, só podem ser desviadas para evitar lesão se houver conhecimento prévio da alteração. ${ }^{4}$ Ainda que a incidência de anomalias vasculares vertebrais seja desconhecida na população geral, uma série de 300 angiografias encontrou incidência de $3 \%{ }^{5}$ Possíveis indicações de exames de imagem vascular antes da cirurgia incluem presença de sintomas de neuralgia occipital e anomalias ósseas congênitas da região craniocervical, como occi- pitalização do atlas. ${ }^{11}$ Ainda assim, Nassr et $\mathrm{al}^{4}$ indicam angiotomografia pré-operatória de rotina para o planejamento de instrumentação cervical superior, particularmente em $\mathrm{C} 1{ }^{4}$ Em última análise, como o cirurgião é responsável pelas complicações associadas aos procedimentos, ele pode decidir quais exames fazer antes da cirurgia. ${ }^{4}$

A variação anatômica apresentada chama a atenção para a importância da dissecção cuidadosa do arco de C1 antes de sua remoção, assim como para o cuidado na coagulação e dissecção de vasos nessa região. Em casos de lesão vascular, alguns pacientes podem se beneficiar do tratamento endovascular. $O$ conhecimento da anatomia vascular da transição crânio-cervical normal e de suas variações permite um diagnóstico topográfico preciso e um planejamento cirúrgico adequado, minimiza complicações, e aprimora os resultados terapêuticos.

\section{Conflitos de Interesse}

Os autores declaram não haver conflitos de interesse.

\section{Referências}

1 Lister JR, Rhoton AL Jr, Matsushima T, Peace DA. Microsurgical anatomy of the posterior inferior cerebellar artery. Neurosurgery 1982;10(02):170-199

2 Fine AD, Cardoso A, Rhoton AL Jr. Microsurgical anatomy of the extracranial-extradural origin of the posterior inferior cerebellar artery. J Neurosurg 1999;91(04):645-652

3 Wallenberg A. Akute Bulbäraffektion (Embolie der Arteria cerebelli post inf sinistra). Archives fur Psychiatry 1895;27:504-540

4 Nassr AN, Swann PP, Huston J III, Abdelfatah MM, Rose PS, Currier BL. Aberrant posterior inferior cerebellar artery injury with $\mathrm{C} 1$ lateral mass screw placement: a case report and review of the literature. Spine J 2014;14(09):e7-e14

5 Tokuda K, Miyasaka K, Abe $\mathrm{H}$, et al. Anomalous atlantoaxial portions of vertebral and posterior inferior cerebellar arteries. Neuroradiology 1985;27(05):410-413

6 Fukuoka T, Takeda H, DemboT, et al. Clinical review of 37 patients with medullary infarction. J Stroke Cerebrovasc Dis 2012;21(07): 594-599

7 Akinduro OO, Baum GR, Howard BM, et al. Neurological outcomes following iatrogenic vascular injury during posterior atlantoaxial instrumentation. Clin Neurol Neurosurg 2016;150:110-116

8 Brinjikji W, Cloft H, Kallmes DF. Anatomy of the posterior inferior cerebellar artery: relevance for C1-C2 puncture procedures. Clin Anat 2009;22(03):319-323

9 Vincentelli F, Caruso G, Rabehanta PB, Rey M. Surgical treatment of a rare congenital anomaly of the vertebral artery: case report and review of the literature. Neurosurgery 1991;28(03):416-420

10 Sharma RR, Parekh HC, Prabhu S, Gurusinghe NT, Bertolis G. Compression of the $\mathrm{C}-2$ root by a rare anomalous ectatic vertebral artery. Case report. J Neurosurg 1993;78(04):669-672

11 Wang S, Wang C, Liu Y, Yan M, Zhou H. Anomalous vertebral artery in craniovertebral junction with occipitalization of the atlas. Spine 2009;34(26):2838-2842 